\title{
Site-Specific Stochastic Propagation Model for Automated RFID Network Planning
}

\author{
A. G. Dimitriou* \\ S. Siachalou ${ }^{\dagger}$ \\ A. Bletsas ${ }^{\ddagger}$ \\ J. Sahalos ${ }^{\S}$
}

\begin{abstract}
In this paper, we propose a model to calculate the probability of successful identification of passive UHF RFID tags in a given environment. The validity of the proposed model is verified by comparison with analytical ray-tracing results and measurements. The model can be included in automated RFID network planning algorithms to evaluate the identification performance of different configurations.
\end{abstract}

\section{INTRODUCTION}

Radio Frequency IDentification (RFID) represent the key enabler of the "Internet of Things" concept [1]. Among the key parameters for the marketpenetration of the technology is to reliably design complex RFID networks that ensure excellent identification performance.

In this context, we present a site-specific stochastic propagation model that is part of an automated RFID site-selection planning problem. In automated planning problems, one should select a subset, among a given a set of possible sites for installing equipment (in our case RFID readers), so that a given cost function is minimized (or maximized) under specific quality of service constraints [2]. As the number of possible sites increases, the exact vector-solution cannot be calculated in reasonable time and search heuristics are invoked, e.g. genetic algorithm.

The typical reader-tag-reader link-model is presented in [3]. Simple path-loss expressions are given. Stochastic propagation-prediction models are presented in [4]-[6]. The authors in [5] test different stochastic models to better describe fading in different indoor situations, e.g. Line Of Sight

\footnotetext{
*Aristotle University of Thessaloniki, Dept. of Electrical and Computer Engineering, 54124, Thessaloniki, Greece, email: antodimi@auth.gr, tel.: +30 6978896350.

†Aristotle University of Thessaloniki, 54124, Thessaloniki, Greece, e-mail: ssiachal@auth.gr, tel.: +30 6977688284 .

$\ddagger$ Technical University of Crete, Dept. of Electronics and Computer Engineering, Akrotiri Campus 73100 Chania, Crete, Greece, e-mail: aggelos@telecom.tuc.gr, tel.: +30 2821037377.

$\S$ Aristotle University of Thessaloniki, 54124, Thessaloniki, Greece, e-mail: sahalos@auth.gr, tel.: +30 2310998161 and University of Nicosia, Dept. of Electrical and Computer Engineering,46 Makedonitissas Avenue 24005 Nicosia, Cyprus, e-mail: sahalos.j@unic.ac.cy, tel.: +357 22841740 .
}

(LOS) or not (NLOS). In [6], the authors evaluate the performance of different diversity schemes, assuming Rayleigh distribution for fading. In [7], a reading region is estimated as the ellipsoid, where the minimum power received from a two-ray model (considering only ground reflection), is greater than the wake-up threshold of the tag. The accuracy of the model depends on the position and direction of the reader's antenna with respect to the environment, as the basic assumption of the model is to consider a reflection from a single surface.

Analytical ray-tracing calculations were presented in [8]-[10], where maximization of the reader's read-region was sought. Different methods to minimize destructive interference effects, due to fading were proposed, including the introduction of a controllable phase shifter in multiple-antennas configurations.

In this paper, we model stochastically any sitespecific environment, though we focus in a typical room. We consider a cubic calculations' grid inside the volume of interest and derive an appropriate Rice probability density function on each gridpoint [11]. The Rice statistics for each calculations' point are derived by an analytic ray-tracing model. Then we calculate the probability of succesfull identification of a tag anywhere in the volume under investigation, for a given "wake-up' tag-threshold. Furthermore, we derive the aggregate identification percentage in the entire volume of interest. We separately analyze identification performance per tag's polarization axis, as well as tag-diversity schemes. Performance of the proposed stochastic model is verified against analytic ray-tracing calculations and measurements.

\section{PROPOSED STOCHASTIC MODEL}

\subsection{Field at the Tag}

Consider a transmitting antenna and a tag inside a room, as shown in Figure 1. The total field at any point in the room can be evaluated as the phasesum of several contributions that have traveled different paths (multiple reflections, scattering). Due the interactions with the environment, each path has suffered different losses and contributes to the total field with a different phase. By analyzing the 
field at the receiver in orthogonal axes, the total field can be written as:

$$
\begin{aligned}
& \mathbf{E}_{\mathbf{t o t}}=\sqrt{\frac{\eta W_{t}}{2 \pi}} \\
&\left(\sum_{j} \frac{\sqrt{G_{t}}\left(\phi_{j}, \theta_{j}\right) A_{j} e^{i\left(2 \omega t+a_{j}+k r_{j}\right)}}{r_{j}} \hat{x}+\right. \\
& \sum_{j} \frac{\sqrt{G_{t}}\left(\phi_{j}, \theta_{j}\right) B_{j} e^{i\left(2 \omega t+b_{j}+k r_{j}\right)}}{r_{j}} \hat{y}+ \\
&\left.\sum_{j} \frac{\sqrt{G_{t}}\left(\phi_{j}, \theta_{j}\right) C_{j} e^{i\left(2 \omega t+c_{j}+k r_{j}\right)}}{r_{j}} \hat{z}\right),
\end{aligned}
$$

where $W_{t}$ is the power of the transmitted carrier, $G_{t}\left(\theta_{j}, \phi_{j}\right)$ is the transmitting antenna's gain at the direction defined by the horizontal and vertical angles $\phi_{j}, \theta_{j}$ respectively, $\eta$ is the free-space impedance, $k=2 \pi / \lambda, \lambda$ is the considered wavelength, $r_{j}$ the path traveled by the $j_{t h}$ contribution and $A_{j}, B_{j}, C_{j}$ size the field of each ray at the corresponding polarization axis, defined by the orthogonal vectors $\hat{x}, \hat{y}, \hat{z}$, respectively.

\subsection{Stochastic Model}

Our target is to evaluate the read-range of a UHF RFID reader that identifies passive RFID tags. Considering the regulated maximum transmitpower constraints of the reader $(\sim 35 \mathrm{dBm}$ EIRP), combined with the significant power needed by the (battery-less) tag to operate $(\sim-18 \mathrm{dBm})$, the reader cannot identify a passive tag blocked behind an object (succcesful identification of a blocked passive RFID tag could occur only at very close proximity with the reader's antenna, $\sim \mathrm{cm}$ ). Hence, a LOS strong path should be present in the reader's reading region. The propagation conditions of the problem justify the selection of the Rice distribution to stochastically describe the expected fading. The Rice probability density function assumes the existance of a strong path (in this case the LOS) and several smaller contributions (multiple reflections). The probability density function is given by:

$$
f(x \mid \nu, \sigma)=\frac{x}{\sigma^{2}} e^{\left(\frac{-\left(x^{2}+\nu^{2}\right)}{2 \sigma^{2}}\right)} I_{0}\left(\frac{x \nu}{\sigma^{2}}\right) .
$$

In $(2), \nu^{2}$ is the power of the LOS path and $2 \sigma^{2}$ is the average power of the other contributions. The cumulative distribution function is given by:

$$
F_{X}(x \mid \nu, \sigma)=1-Q_{1}\left(\frac{\nu}{\sigma}, \frac{x}{\sigma}\right), \text { where }
$$

$Q_{1}$ is the Marcum Q-function.

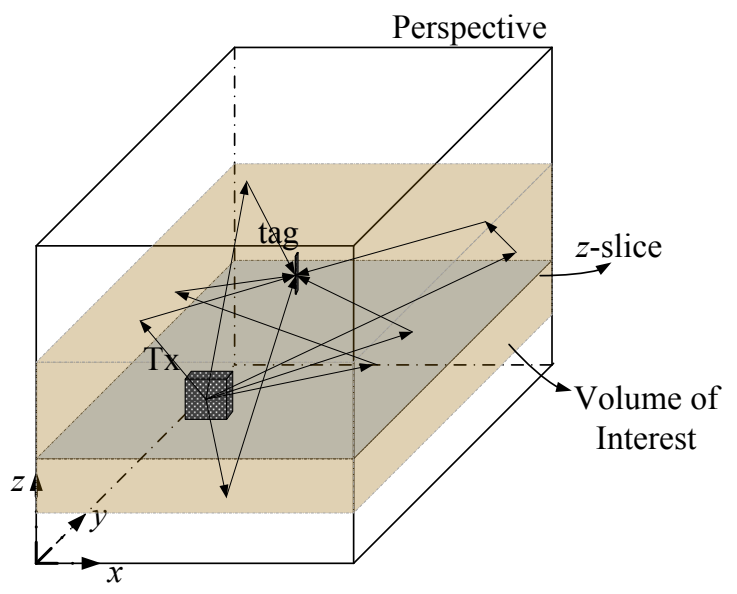

Figure 1: Representation of the modeled geometry.

In order to evaluate the probability of successful identification on a given point, we need to separately calculate the LOS power and the average power of all other contributions at the location of interest. We expect the phases of the multiply reflected rays to be uncorrelated, because of the large path-length differences of the multipath components, with respect to $\lambda$; in (1), $2 \pi\left(r_{m}-r_{m-1}\right) / \lambda \gg$ $2 \pi, \forall m$ and $r_{m}-r_{m-1} \neq r_{j}-r_{j-1}, \forall m \neq j$. We consider the phases of the multipath components as random variables identically and independently distributed, uniformly over $[0,2 \pi]$. Under these assumptions, it can be shown that the average received power due to the terms in (1), except for the LOS contribution, is given by the appropriate sum of the square of the magnitudes, [12], of each contribution. The average power on a receiving antenna with unity gain per polarization axis is the given by:

$$
\begin{aligned}
P_{x}=\frac{\eta W_{t}}{2 \pi} \sum_{j \neq \mathrm{LOS}} \frac{G_{t}\left(\phi_{j}, \theta_{j}\right) A_{j}^{2}}{r_{j}}, \\
P_{y}=\frac{\eta W_{t}}{2 \pi} \sum_{j \neq \mathrm{LOS}} \frac{G_{t}\left(\phi_{j}, \theta_{j}\right) B_{j}^{2}}{r_{j}}, \\
P_{z}=\frac{\eta W_{t}}{2 \pi} \sum_{j \neq \mathrm{LOS}} \frac{G_{t}\left(\phi_{j}, \theta_{j}\right) C_{j}^{2}}{r_{j}} .
\end{aligned}
$$

We consider a calculations' grid with $M$ points in the volume of interest. A passive tag is considered succesfully identified if the power that reaches the tag is greater than its "wake-up" threshold $\gamma$. On each position of the grid $l$, we calculate the probability that a tag is succesfully identified as:

$$
P_{l}(X \geq \gamma)=1-F_{X}\left(\gamma \mid \nu_{l}, \sigma_{l}\right)
$$




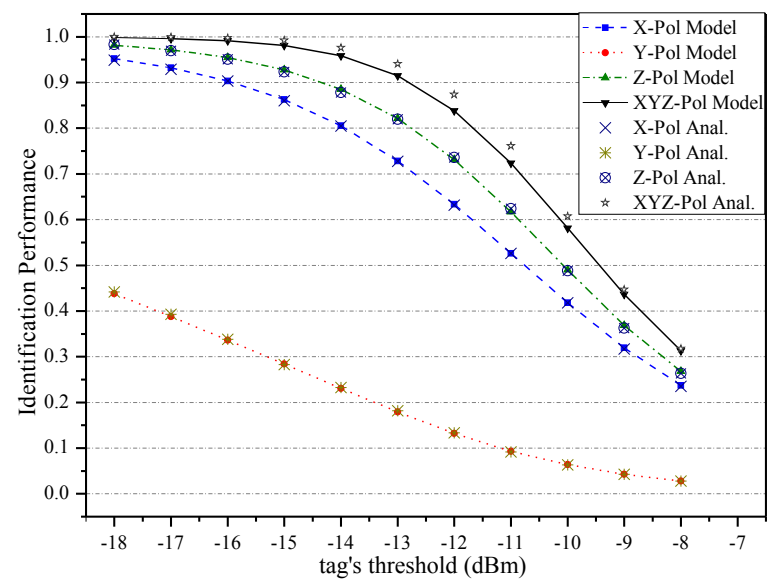

Figure 2: Comparison between stochastic model and analytical ray-tracing predictions.

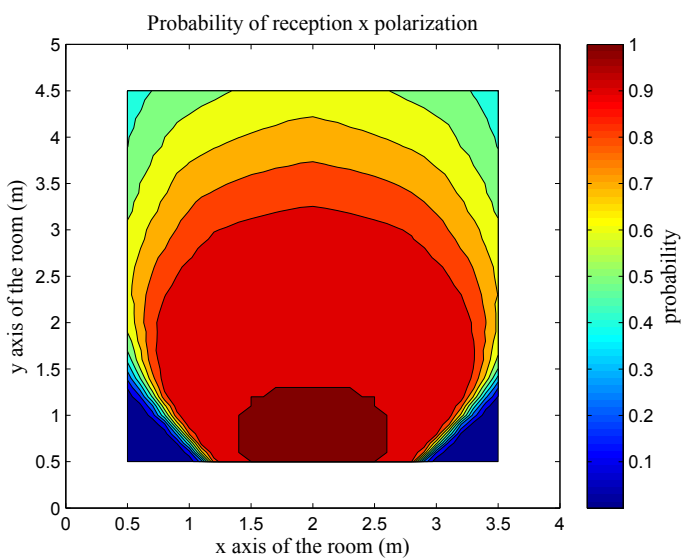

Figure 3: Probability of successful identification of Xpolarized tags for $\gamma=-14 \mathrm{dBm}$.

where $F_{X}\left(\gamma \mid \nu_{l}, \sigma_{l}\right)$ is given in (3); $\nu_{l}^{2}$ is the power of the LOS path on the polarization axis of the $\operatorname{tag}$ and $2 \sigma_{l}^{2}$ is the power of the multiply reflected contributions, calculated by applying (4) for the tag's polarization axis. As a consequence, fading and the associated identification-probability is separately evaluated at each location in the volume of interest depending on the site-specifically calculated parameters of the distribution. Let $U(\gamma)$ represent the percentage of the volume of interest $V$, where succesful identification of passive tags is accomplished. $U(\gamma)$ is evaluated as:

$$
U(\gamma)=\int_{V} P_{d V}(X \geq \gamma) d V=\frac{1}{V} \sum_{l=1}^{M} P_{l}(X \geq \gamma) d V_{l}
$$

For a cubic calculations' grid, with equal spacing among grid points, (6) reduces to $U(\gamma)=$ $\sum_{l=1}^{M} P_{l}(X \geq \gamma) / M$.

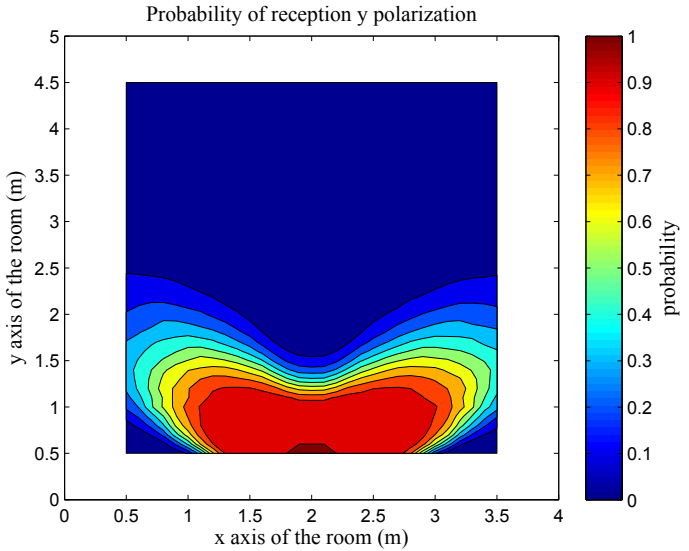

Figure 4: Probability of successful identification of Ypolarized tags for $\gamma=-14 \mathrm{dBm}$.

\section{RESULTS}

We consider a 7 dBic-gain circularly polarized (along the $\mathrm{X}$ and $\mathrm{Z}$ axes) reader-antenna, placed on the center of a $4 \mathrm{~m}$-length wall, inside a $4 \mathrm{~m} \times 5 \mathrm{~m} \times 3 \mathrm{~m}$ room, as demonstrated in Figure 1. We calculate the probability of successful identification for increasing tag's threshold $\gamma$ from $-18 \mathrm{dBm}$ to $-8 \mathrm{dBm}$, by applying (4)-(5); then we calculate the percentage of successful identification in the volume of interest from (6) for all polarizations and tags' diversity. The results are compared with the corresponding results from an analytical ray-tracing model, where the percentage of succesfully identified tags in the same volume of interest is calculated. The results are summarized in Figure 2. Very good agreement is recorded for all polarizations and thresholds.

The probability of identification for $\mathrm{X}$ and $\mathrm{Y}$ polarized tags, considered $1.1 \mathrm{~m}$ above the ground, is demonstrated in Figures 3-4. Notice the correctly calculated reduced probability of identification for Y polarized tags, as the reader's antenna is polarized on the $\mathrm{X}$ and $\mathrm{Z}$ axes.

Comparison of the performance of the proposed model with measurements conducted in a $3 \mathrm{~m} \times 3.5 \mathrm{~m} \times 3 \mathrm{~m}$ room are given in Figures $5-6$. The transmitted power from the reader was reduced from $30 \mathrm{dBm}$ to $15 \mathrm{dBm}$. Details on the measurements can be found in [9]. Very good agreement was again recorded.

\section{CONCLUSIONS}

In this work, we presented a stochastic model that exploits site-specific data to evaluate the identification performance of RFID systems, employing passive UHF tags. Performance of the model was 

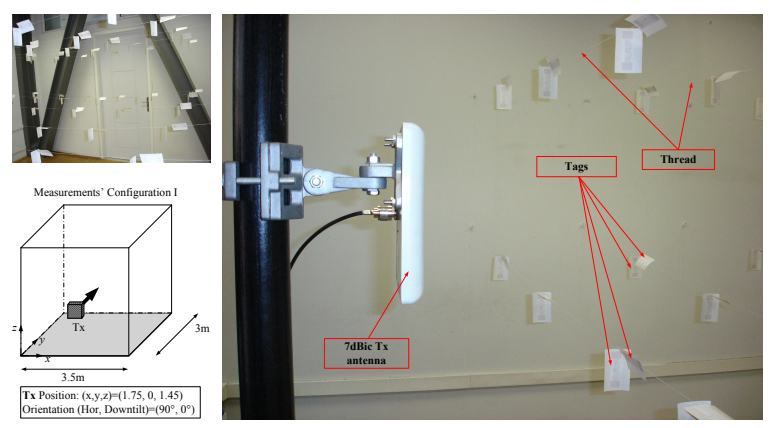

Figure 5: Measurements of orthogonally polarized tags in a room.

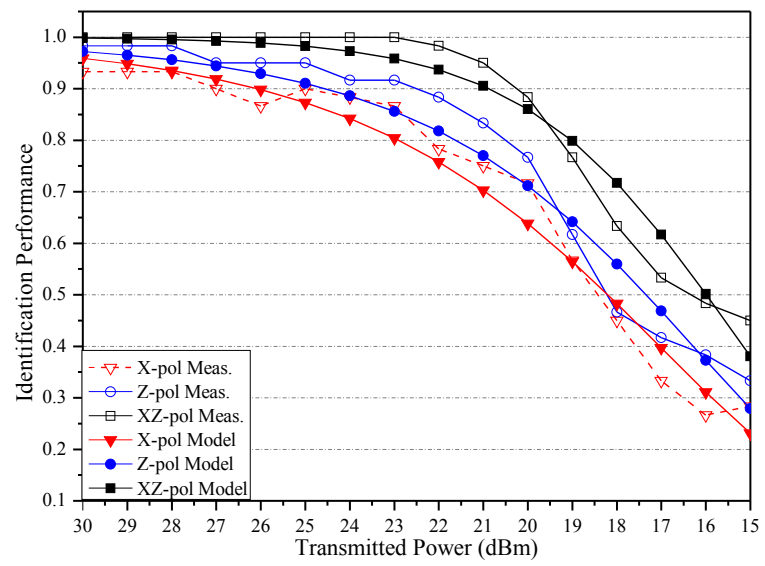

Figure 6: Comparison between model estimations and measurements in a room.

validated from measurements and comparison with analytical solutions. Further work should focus on acceleration techniques, e.g. reducing the number of multipath components involved.

\section{Acknowledgments}

This research has been co-financed by the European Union (European Social Fund-ESF) and Greek national funds through the Operational Program "Education and Lifelong Learning" of the National Strategic Reference Framework (NSRF) - Research Funding Program: THALES. Investing in knowledge society through the European Social Fund.

\section{References}

[1] D. M. Dobkin, "The RF in RFID: Passive UHF RFID in Practice", Oxford, Boston, Newnes (Elsevier), 2007.

[2] A. Bahri, S. Chamberland, "On the wireless local area network design problem with performance guarantees", Elsevier Computer Networks, vol. 48, no. 6, pp. 856-866, Aug. 2005.
[3] P. V. Nikitin, K. V. S. Rao, "Antennas and Propagation in UHF RFID Systems", 2008 IEEE International Conference on RFID, pp. 277-288.

[4] J. D. Griffin, and G. D. Durgin, "Complete Link Budgets for Backscatter-Radio and RFID Systems", IEEE Antennas and Propagation Magazine, vol. 51, no. 2, pp. 11-25, April 2009.

[5] A. Lazaro., D. Girbau, and D. Salinas, "Radio Link Budgets for UHF RFID on Multipath Environments", IEEE Transactions on Antennas and Propagation, vol. 57, no. 4, pp. 1241-1251, April 2009.

[6] J. D. Griffin, and G. D. Durgin, "Gains For RF Tags Using Multiple Antennas", IEEE Transactions on Antennas and Propagation, vol. 56, no. 2, pp. 563-570, February 2008.

[7] G. Marrocco, E. Di Giampaolo, and R. Aliberti., "Estimation of UHF RFID Reading Regions in Real Environments", IEEE Antennas and Propagation Magazine, vol. 51, no 6, pp. 44-57, December 2009.

[8] A. G. Dimitriou, A. Bletsas and J. N. Sahalos, "On Efficient UHF RFID Coverage inside a Room", European Conference on Antennas and Propagation, Barcelona, 12-16 April 2010.

[9] A. G. Dimitriou, A. Bletsas and J. N. Sahalos, "Room Coverage Improvements of UHF RFID with Commodity Hardware", IEEE Antennas and Propagation Magazine, vol. 53, no. 1, February 2011.

[10] A. G. Dimitriou, A. Bletsas, A. Polycarpou, and J. N. Sahalos, "Theoretical Findings and Measurements on Planning a UHF RFID System inside a Room", Radioengineering Journal - Towards EuCAP 2012: Emerging Materials, Methods, and Technologies in Antennas and Propagation, vol. 20, no. 2, pp. 387-397, June 2011.

[11] S. O. Rice, "Mathematical Analysis of Random Noise", Bell System Technical Journal, vol. 24, pp. 46156, 1945.

[12] T. S. Rappaport, "Mobile Radio Propagation: Small Scale Fading and Multipath" in Wireless Communications Principles and Practice, 2nd Ed. Prentice Hall, 2001, ch. 5. 